\title{
Balistic magnetoresistance in nanocontacts electrochemically grown between macro- and microscopic ferromagnetic electrodes
}

\author{
N. García, G. G. Qiang, and I. G. Savelieva) \\ Laboratorio de Física de Sistemas Pequeños y Nanotecnología CSIC, Serrano 144 E-28006 Madrid Spain
}

(Received 8 August 2001; accepted for publication 11 January 2002)

\begin{abstract}
Our results prove the local origin of magnetoresistance in electrochemically deposited $\mathrm{Ni}$ nanocontacts. Experiments have been done using a complex setup for both in situ growth and ballistic magnetoresistance (BMR) measurements. Nanocontacts have been grown between two macroscopic $\mathrm{Ni}$ wires. In situ experiments with variation of the nanocontact diameter from 3 to 20 $\mathrm{nm}$ have been done using the same pair of wires. BMR values from $0.5 \%$ to $100 \%$ have been observed but no correlation of BMR value with the sample resistance, i.e., with the nanocontact cross section, has been found. These results show that the BMR in the nanometric size contact is determined by local geometrical and magnetic structures near the nanocontact rather than by the contact cross section itself. The hypothesis of existence of the intrinsic nonmagnetic dead layer in the ferromagnetic nanocontact is proposed to account for the BMR properties of the nanometric size contacts. Additionally, we report a BMR value of $200 \%$ in a $\mathrm{Ni}$ nanocontact (5 nm diameter) electrochemically grown between two nonmagnetic macroscopic gold wires. An external magnetic field has been used during the electrochemical deposition to fix the easy magnetic axis of the deposited Ni layer. (C) 2002 American Institute of Physics. [DOI: 10.1063/1.1459108]
\end{abstract}

Ballistic magnetoresistance (BMR) in ferromagnetic atomic size (less than $1 \mathrm{~nm}$ diameter) nanocontacts were first reported in 1999. ${ }^{1}$ The physical origin of the large magnetoresistance (MR) values (up to $300 \%$ at room temperatures) is a modification of the spin-dependent transparency of the nanocontact by the external magnetic field that changes the orientation of magnetization in an area of a few nanometers near the contact. ${ }^{2}$ The BMR is a local effect, i.e., local magnetization near the contact plays the dominant role. Atomic size contacts are stable for a few minutes only, which impairs technological applications. Subsequent investigations have shown very large BMR (up to $700 \%$ ) at room temperatures for nanometric size (1-100 nm diameter) contacts. ${ }^{3,4}$ These nanocontacts were electrochemically grown between two ferromagnetic wires and they are stable for days. Stable BMR structures can successfully compete with giant $\mathrm{MR}^{5}$ and tunnel $\mathrm{MR}^{6}$ structures for applications as local magnetic sensors or as reading magnetic heads. An important question, which is very significant for practical applications, is the role of the bulk ferromagnetic electrodes. The use of large ferromagnetic electrodes $(5 \mathrm{~mm}$ long and $0.1 \mathrm{~mm}$ diameter $\mathrm{Ni}$ wire) raises the question of the possible role of bulk magnetic effects, such as static magnetic forces or magnetostriction. On the other hand, the structures with nanocontacts can not function as local sensors if it is necessary to modify the magnetization of the bulk electrodes to get a magnetoresistive response. The experiments reported here prove the local origin of the BMR in electrochemically deposited nanocontacts. It is clear that massive metallic electrodes are playing the role of radiators stabilizing the thermal balance of the system.

An experimental setup has been designed for this pur-

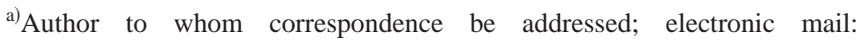
IGS@fsp.csic.es
}

pose. With this setup, we can do in situ MR meaurements while the sample is within the growing solution. Ni nanocontacts have been produced electrochemically in a saturated $\mathrm{NiSO}_{4}$ aqueous solution. The electrochemical cell is placed inside the electromagnet, which provides magnetic fields up to $4.5 \mathrm{kOe}$. An electrochemical controller based on a bipotentiostat (Model AFCBP1, Pine Instruments Company) sets the electrochemical potential at the working electrode and collects values of dc current between the working electrode and the solution. A measuring system provides a small ac current between the working electrodes and collects data of the resistance $R_{c}$ of the nanocontact growing between them. A computer controls all systems, provides the negative feedback during electrochemical growth and stops all electrochemical process during magnetoresistance measurements. The contact resistance is measured by using either a standard lock-in technique or normal dc method, in both cases a current of 5 to $100 \mu \mathrm{A}$ was allowed to flow through the contact. Usually it takes $10 \mathrm{~min}$ to get the data for one experimental MR curve.

To demonstrate the local origin of nanocontact MR, we have made two series of experiments. For the first one, we have prepared the sample in the same manner as described in previous papers. ${ }^{4}$ Two Ni wires $(0.1 \mathrm{~mm}$ diameter and $5 \mathrm{~mm}$ length) are fixed at a dielectric substrate (see inset of Fig. 1). The top of the vertical electrode (WE1 in Inset Fig. 1) is placed at a distance about $20 \mu \mathrm{m}$ to the central part of the horizontal electrode (WE2). A dielectric glue covers the sample except the top part of the vertical electrode and the central part of the horizontal electrode. Deposition of $\mathrm{Ni}$ takes place on the open part of WE1, which is closest to WE2. When a contact between WE1 and WE2 is detected, a feedback system starts to function and provides a predetermined value of resistance for the nanocontact. After each MR measurement, we set a new value $R_{\text {set }}$ and restart the elec- 


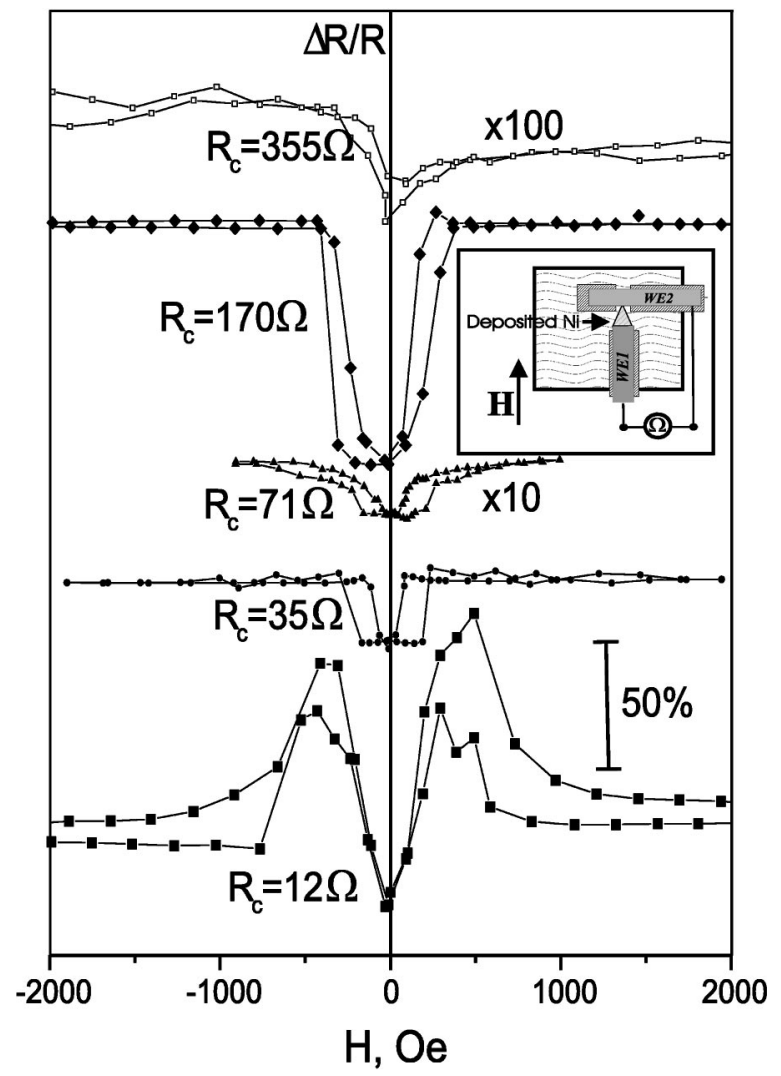

FIG. 1. Magnetic field dependencies of normalized MR $\Delta R / R$ $\equiv R(H) / R_{c}-1$ corresponds to five different values of contact resistance $R_{c}$, which are printed near the curves. All curves are presented with the same scale (see bar corresponding to 50\% MR) but with vertical offset. Only data corresponding to $71 \Omega$ resistance have been multiplied by 10 , and data corresponding to $R_{c}=355 \Omega$ have been multiplied by 100 . All data have been obtained at one in situ experiment. Sample is schematically presented in inset. Nanocontact have been electrochemically grown between two $\mathrm{Ni}$ wire and have been modified to study dependence of MR on the nanocontact cross sections.

trochemical process. It should be emphasized that the existing contact is not destroyed and the wires remain unchanged. The sample configuration is the same; we just change the cross section of the nanocontact using electrochemical etching or deposition. In this manner, we have investigated several nanocontacts with different cross sections but with the same bulk electrodes. If bulk magnetic effects determine MR of the nanocontact then we should obtain the same type and the same value of MR for all these measurements.

Experimental results are presented in Fig. 1. One can see the magnetic field dependence of the normalized MR $\Delta R / R \equiv R(H) / R_{c}-1$ corresponding to five different values of contact resistance $R_{c}$, printed near the curves. All curves are presented with the same scale but with vertical offset. Only the data corresponding to $71 \Omega$ resistance have been multiplied by 10 , and the data for the case of the highest resistance $R_{c}=355 \Omega$ have been multiplied by 100 . The maximum values of MR $105 \%$ and $95 \%$ are found for $R_{c}$ $=12 \Omega$ and $170 \Omega$, respectively. Nearly $30 \%$ MR was observed for a resistance $35 \Omega$. Very small changes of resistance of $2.2 \%$ and $0.5 \%$ are observed for samples of $R_{c}$ $=71 \Omega$ and $355 \Omega$, respectively. These very different values of MR obtained for the same bulk electrodes clearly demonstrate that BMR in ferromagnetic nanocontacts is not deterDownloaded 15 Feb 2010 to 161.111.180.191. Redistribution subject mined by bulk magnetic effects such as magnetostriction and magnetic forces.

Contrarily to the results obtained for mechanically produced atomic size contacts, ${ }^{1,2}$ we do not observe a clear dependence of the MR value with the sample resistance. The most common point of view is that the resistance of quantum $^{7}$ and classical ${ }^{8}$ point-contacts is determined by the smallest diameter of the constriction or by the contact diameter, and that the geometrical details on the vicinity of the contact or the form of the contact are irrelevant. But, as it was demonstrated theoretically, ${ }^{9,10}$ the geometrical details of the vicinity of the contact strongly influence the width of the domain wall captured by the nanocontact and, therefore, ${ }^{2}$ the contact form determines the transparency of the domain wall for spin-polarized electrons. It is important to note that the model of a constrained domain wall developed by Bruno ${ }^{9}$ can not account for the very big BMR in the nanometer size contacts. Detailed calculations ${ }^{10}$ show that the domain wall in the constriction is not one dimensional, as Bruno believed, and that the domain wall width can not be less than the contact diameter. Electron scattering by this domain wall is negligible for nanometers size nanocontact. ${ }^{2,11}$ Therefore, to explain a BMR value up to $700 \%$ (Ref. 4) in an electrochemically grown Ni nanocontact we have to assume that the nanocontact, contains an intrinsic "dead layer" of nonmagnetic material. In this case, the exchange interaction is negligible and the effective wall width is equal the dead layer thickness, which can be of atomic dimension. This assumption is quite reasonable since many experiments indicate that magnetic films are composed of magnetic grains with nonmagnetic grain boundaries. ${ }^{12}$ In the insets of the Fig. 2, we present electron-microscope images of the surface of Ni layers electrodeposited at standard conditions $\mathrm{H}=0$ [Fig. 2(a)] and at the external magnetic field $2.5 \mathrm{kOe}$ [Fig. 2(b)]. The micrograin structure can be seen clearly in both images, but the surface of the film is smoother if electrochemical deposition has been done in the presence of magnetic field.

When we change the sample resistance during the electrochemical growth, we control only the diameter of the nanocontact. But, for sure, the periodically repeated processes of etching and deposition can vary the shape of the $\mathrm{Ni}$ bridge and the properties of the intrinsic dead layer in the nanocontact. These uncontrolled small variations of the contact geometry or local magnetic structures near the contact can influence strongly the MR of the nanocontact with different diameters, as presented in Fig. 1.

We note that the MR curve for the nanocontact with the largest diameter (smallest $R_{c}$ ) includes both positive and negative MR. The transition from positive to negative MR takes place at around 300 Oe. Negative MR may have two possible causes, the first being that several domains are sweeping across the contact, ${ }^{3}$ and the second that the magnetization on the opposite side of the contact becomes oriented by the external field. Both origins point to the fact that the effect is a highly local effect, i.e., the behavior is mostly determined by the magnetization configuration in the proximity of the contact rather than in the bulk electrodes.

To eliminate any bulk magnetic effects, we have used in the second experiment $\mathrm{Cu}$ or $\mathrm{Au}$ electrodes instead of ferromagnetic Ni electrodes. To produce the ferromagnetic nanoto AIP license or copyright; see http://apl.aip.org/apl/copyright.jsp 


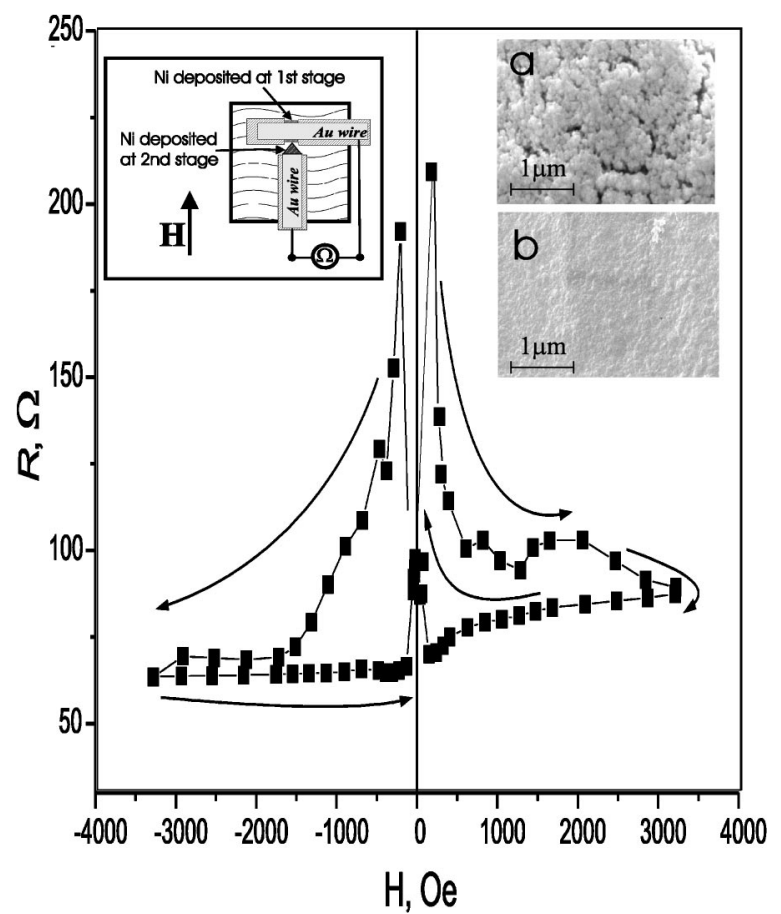

FIG. 2. MR of the Ni-Ni nanocontact grown between the two gold bars. Schema of the sample is presented in inset. First stage deposition of the $\mathrm{Ni}$ on the horizontal wire was made at magnetic field $4 \mathrm{kOe}$ parallel to axe of this bar. MR measurements have been done at magnetic field, which is parallel to another wire. Electron-microscope images of the electrodeposited Ni layers are presented in inset (a) and (b). In both cases, electrodeposition of $\mathrm{Ni}$ on the gold has been done at the same condition, just in the case (b) external magnetic field $2.5 \mathrm{kOe}$ have been applied during deposition.

contact, the electrochemical deposition has been made in two stages. At a first stage, we deposited a Ni layer (several micron thickness) on the open part of the horizontal electrode (see inset at Fig. 2). The second stage was the same as described herein. Deposition has been done on the top of the vertical electrode to get a $\mathrm{Ni}-\mathrm{Ni}$ nanocontact of a predetermined resistance. The nanocontact is formed between microscopic (a few microns) pieces of Ni. In this case, elongation due to magnetostriction is less than $10^{-2} \mathrm{~nm}$, which can not influence the contact of several nanonmeters diameter.

We observed very small MR (less 5\%) for most of the samples prepared in this manner. We believe that this may be due to a chaotic orientation of the magnetic structure at both sides of the contact. Contrarily to the case of structures based on $\mathrm{Ni}$ wires, here there are no bulk magnetic wires to provide a strong magnetic anisotropy that fixes the easy magnetic axes at the vicinity of the nanocontact. Electrochemical deposition of $\mathrm{Ni}$ on the nonmagnetic substrate provides micrograin layers with chaotically oriented magnetic anisotropy. Therefore, we have no preferred directions at the vicinity of the nanocontact and the external magnetic field can simultaneously change magnetization at either side of the nanocontact. As a result, the transparency of the nanocontact will be changed weakly by the magnetic field and MR should be very small. To solve this problem, we have modified the first stage of Ni deposition. We have deposited nickel at the middle part of the horizontal Au bar under an applied magnetic field of 4000 Oe along this bar. We hoped that the external magnetic field would fix magnetic anisotropy and magnetization of the film grown. During MR measurements, the magnetic field is along the perpendicular bar and perpendicular to the easy axis of the Ni film at the horizontal electrode. This magnetic field can not change the magnetization of the horizontal electrode, but could easily change the magnetic structures near the contact at the vertical bar. In Fig. 2 we present experimental MR measurements for this sample, with a nanocontact of $5 \mathrm{~nm}$ diameter, showing values about $200 \%$ MR. These clearly demonstrate that by using an external magnetic field, we can control and fix the orientation of the magnetic easy axis during electrochemical deposition of a magnetic film on a nonmagnetic substrate.

Finally, we report values of $200 \%$ BMR in electrochemically deposited ferromagnetic nanocontacts grown between microscopic pieces of Ni. In situ experiments have demonstrated that macroscopic magnetic effects are not relevant in determining the BMR of the electrochemically deposited $\mathrm{Ni}$ nanocontacts, but that BMR strongly depends on the local magnetic structure near the contact and the local contact geometry rather than on the contact cross section. Hypothesis of the intrinsic nonmagnetic dead layer in the ferromagnetic nanocontact has been proposed to describe the BMR for nanometric size nanocontacts.

This work has been supported by EU contract IST-200026011. One of the authors (I.G.S.) is supported by a NATO science program. Thanks are extended to Professor J. A. Rausell Colom for his help.

${ }^{1}$ N. García, M. Muñoz, and Y.-W. Zhao, Phys. Rev. Lett. 82, 2923 (1999).

${ }^{2}$ G. G. Cabrera and L. M. Falicov, Phys. Status Solidi B 61, 539 (1974); G. Tatara and H. Fukuama, Phys. Rev. Lett. 78, 3773 (1997); G. Tatara, Y.-W. Zhao, M. Muñoz, and N. García, ibid. 83, 2030 (1999).

${ }^{3}$ N. García, I. G. Saveliev, Y.-W. Zhao, and A. Zlatkine, J. Magn. Magn. Mater. 214, 7 (2000); N. García, H. Rohrer, I. G. Saveliev, and Y.-W. Zhao, Phys. Rev. Lett. 85, 3053 (2000).

${ }^{4}$ N. García, M. Muñoz, G. G. Qian, H. Rohrer, I. G. Saveliev, and Y. W. Zhao, Appl. Phys. Lett. (submitted); N. García, M. Muñoz, V. V. Osipov, E. V. Ponizovskaya, G. G. Qian, I. G. Saveliev, and Y.-W. Zhao, J. Magn. Magn. Mater. (to be published).

${ }^{5}$ M. N. Baibich, J. M. Broto, A. Fert, F. Nguyen Van Dau, F. Petroff, P. Eitenne, G. Creuzet, A. Friederich, and J. Chazelas, Phys. Rev. Lett. 61, 2472 (1988); G. Binasch, P. Grünberg, F. Saurenbach, and W. Zinn, Phys. Rev. B 39, 4828 (1989).

${ }^{6}$ J. S. Moodera, L. R. Kinder, T. M. Wong, and R. Meservey, Phys. Rev. Lett. 74, 3273 (1995); S. Tehrani, J. M. Slaughter, E. Chen, M. Durlam, J. Shi, and M. DE Herrera, IEEE Trans. Magn. 35, 2814 (1999).

${ }^{7}$ A. G. Jansen, A. P. van Gelder, and P. Wider, J. Phys.: Condens. Matter 13, $6073(1980)$

${ }^{8}$ G. Wexler, Proc. Phys. Soc. London 89, 927 (1966).

${ }^{9}$ P. Bruno, Phys. Rev. Lett. 83, 2425 (1999).

${ }^{10}$ V. A. Molyneux, V. V. Osipov, and E. V. Ponizovskaya (unpublished).

${ }^{11}$ N. Giordano and J. D. Mounier, Physica D 194, 1009 (1994); K. Hought and N. Giordano, Phys. Rev. B 51, 9855 (1995).

${ }^{12}$ F. Gordon and J. Hughes, J. Appl. Phys. 49, 5306 (1983). 